PROCEEDINGS OF THE

AMERICAN MATHEMATICAL SOCIETY

Volume 136, Number 8, August 2008, Pages 2803-2814

S 0002-9939(08)09352-0

Article electronically published on March 21, 2008

\title{
AN ULTRAMETRIC VERSION OF THE MAILLET-MALGRANGE THEOREM FOR NONLINEAR $q$-DIFFERENCE EQUATIONS
}

\author{
LUCIA DI VIZIO \\ (Communicated by Carmen C. Chicone)
}

\begin{abstract}
We prove an ultrametric $q$-difference version of the MailletMalgrange theorem on the Gevrey nature of formal solutions of nonlinear analytic $q$-difference equations. Since $\operatorname{deg}_{q}$ and $\operatorname{ord}_{q}$ define two valuations on $\mathbb{C}(q)$, we obtain, in particular, a result on the growth of the degree in $q$ and the order at $q$ of formal solutions of nonlinear $q$-difference equations, when $q$ is a parameter. We illustrate the main theorem by considering two examples: a $q$-deformation of "Painlevé II", for the nonlinear situation, and a $q$-difference equation satisfied by the colored Jones polynomials of the figure 8 knots, in the linear case.

We also consider a $q$-analog of the Maillet-Malgrange theorem, both in the complex and in the ultrametric setting, under the assumption that $|q|=1$ and a classical diophantine condition.
\end{abstract}

\section{INTRODUCTION}

In 1903, E. Maillet Mai03 proved that a formal power series solution of an algebraic differential equation is Gevrey. B. Malgrange Mal89 generalized and made more precise Maillet's statement in the case of an analytic nonlinear differential equation. Finally C. Zhang Zha98 proved a $q$-difference-differential version of the Maillet-Malgrange theorem. In the meantime a Gevrey theory for linear $q$-difference-differential equations has been largely developed; $c f$. for instance Ram78, Béz92b], [NM93, [FJ95.

In this paper we prove an analogue of the Maillet-Malgrange theorem for ultrametric nonlinear analytic $q$-difference equations, under the assumption $|q| \neq 1$. It generalizes to nonlinear $q$-difference equations a theorem of Bézivin and Boutabaa; cf. BB92. The proof follows Mal89.

The same technique allows us to prove a Maillet-Malgrange theorem for $q$ difference equations when $|q|=1$, both in the complex and in the ultrametric setting, under a classical diophantine hypothesis: this result generalizes the main result of [Béz92a] and answers a question asked therein. Notice that the problem of nonlinear differential equation in the ultrametric setting is treated in [SSa, SS81, [SSb], where a $p$-adic avatar of diophantine conditions on the exponents is also assumed.

Received by the editors November 13, 2006 .

2000 Mathematics Subject Classification. Primary 33E99, 39A13.

(C)2008 American Mathematical Society Reverts to public domain 28 years from publication 
One of the reasons that makes the ultrametric statement interesting is the possible application to the case when $q$ is a parameter ( $c f$. \$2 below). For instance, when $q$ is a parameter, Corollary 5 ( $c f$. below) becomes:

Theorem 1. Suppose that we are given a nontrivial algebraic nonlinear q-difference equation

$$
F\left(q, x, y(x), \ldots, y\left(q^{n} x\right)\right)=0,
$$

i.e. $F\left(q, x, w_{0}, \ldots, w_{n}\right) \in \mathbb{C}\left[q, x, w_{0}, \ldots, w_{n}\right]$ nonidentically zero, with a formal solution $y(x)=\sum_{h>0} y_{h} x^{h} \in \mathbb{C}(q)[[x]]$. Then there exist nonnegative numbers $s, s^{\prime}$ such that

$$
\limsup _{h \rightarrow \infty} \frac{1}{h}\left(\operatorname{deg}_{q} y_{h}-s \frac{h(h-1)}{2}\right)<+\infty
$$

and

$$
\limsup _{h \rightarrow \infty} \frac{1}{h}\left(\operatorname{ord}_{q} y_{h}-s^{\prime} \frac{h(h-1)}{2}\right)>-\infty
$$

We could give a more precise statement in which $1 / s$ and $-1 / s^{\prime}$ (with the convention $1 / 0=+\infty)$ are slopes of the Newton polygon of the linearized $q$-difference operator of $F\left(q, x, y(x), \ldots, y\left(q^{n} x\right)\right)=0$ along $y(x)$ (cf. Theorem 6).

In classical literature on special functions, $q$ is frequently a parameter. Basic hypergeometric equations are the most classical example in the linear case, while the $q$-analogue of Painlevé equations are a nonlinear example that has been largely studied in the last years 1 This ultrametric "q-adic" approach to the study of a family of functional equations depending on a parameter is peculiar to $q$-difference equations.

\section{ULtRAMETRIC $q$-ANALOG \\ OF The MAILlet-Malgrange TheOrem For $|q| \neq 1$}

Let $\Omega$ be a complete ultrametric valued field, equipped with the ultrametric norm | |, and let $q \in \Omega$ be an element of norm strictly greater than 12

1.1. Digression on the linear case. We denote by $\Omega\{x\}$ the ring of germs of analytic functions at 0 with coefficients in $\Omega$, i.e. the convergent elements of $\Omega[[x]]$. To the linear $q$-difference equation

$$
\mathcal{L} y(x)=\sum_{i=0}^{n} a_{i}(x) y\left(q^{i} x\right)=0,
$$

with $a_{i}(x) \in \Omega\{x\}$, we can attach the Newton polygon

$$
N_{q}(\mathcal{L})=\text { convex envelop }\left(\bigcup_{i=0}^{n}\left\{(i, h): h \geq \operatorname{ord}_{x=0} a_{i}(x)\right\}\right) .
$$

Of course the polygon $N_{q}(\mathcal{L})$ has a finite number of finite sides, with rational slopes, plus two infinite vertical sides. We adopt the convention that the right vertical side has slope $+\infty$ and the left one has $-\infty$.

Bézivin and Boutabaa have proved the following result:

\footnotetext{
${ }^{1}$ For some examples of formal solutions of Painlevé equations $c f$. for instance RGTT01.

${ }^{2}$ We could have chosen the opposite convention $|q|<1$, which leads to analogous statements.
} 
Theorem 2 ([BB92]). Let $g(x) \in \Omega\{x\}$ and $y(x)=\sum_{h \geq 0} y_{h} x^{h} \in \Omega[[x]]$ be such that $\mathcal{L} y(x)=g(x)$. Then either $y(x) \in \Omega\{x\}$ or there exists a positive slope $r \in$ ] $0,+\infty\left[\right.$ of $N_{q}(\mathcal{L})$ such that

$$
\sum_{h \geq 0} \frac{y_{h}}{q^{\frac{h(h-1)}{2 r}}} x^{h}
$$

is a convergent nonentire series.

1.2. Statement of the main result. Consider an analytic function at 0 of $n+2$ variable, i.e. a power series

$$
F\left(x, w_{0}, w_{1}, \ldots, w_{n}\right)=\sum_{k, k_{0}, \ldots, k_{n}>0} A_{k, k_{0}, \ldots, k_{n}} x^{k} w_{0}^{k_{0}} \cdots w_{n}^{k_{n}} \in \Omega\left[\left[x, w_{0}, w_{1}, \ldots, w_{n}\right]\right],
$$

such that

$$
\limsup _{k+\sum_{i=0}^{n} \sup _{k_{i} \rightarrow \infty}}\left|A_{k, k_{0}, \ldots, k_{n}}\right|^{\frac{1}{k+\sum_{i=0}^{n} k_{i}}}<+\infty .
$$

Remark that we have assumed, with no loss of generality, that $F(0, \ldots, 0)=0$. We are interested in studying formal solutions of the nonlinear analytic q-difference equation

$$
F\left(x, \varphi(x), \varphi(q x), \ldots, \varphi\left(q^{n} x\right)\right)=0 .
$$

To simplify notation for any $\varphi \in \Omega[[x]]$ we set $\Phi=\left(\varphi(x), \varphi(q x), \ldots, \varphi\left(q^{n} x\right)\right)$, and we denote by $\sigma_{q}$ the usual $q$-difference operator acting on $\Omega[[x]]$ :

$$
\begin{aligned}
\sigma_{q}: \quad \Omega[[x]] & \longrightarrow \Omega[[x]], \\
\varphi(x) & \longmapsto \varphi(q x) .
\end{aligned}
$$

For any formal power series $\varphi(x) \in \Omega[[x]]$, such that $\varphi(0)=0$, let $\mathcal{F}_{\varphi}$ be the linearized q-difference operator of $F$ along $\varphi$ :

$$
\mathcal{F}_{\varphi}=\sum_{i=0}^{n} \frac{\partial F}{\partial w_{i}}(x, \Phi) \sigma_{q}^{i}
$$

The operator $\mathcal{F}_{\varphi}$ being linear, we can define its Newton polygon $N_{q}\left(\mathcal{F}_{\varphi}\right)$ in the usual way ( $c f$. equation (1) $)$. We want to prove that, for a solution $\varphi(x)$ of (2), the positive slopes of $N_{q}\left(\mathcal{F}_{\varphi}\right)$ are linked to the $q$-Gevrey order of $\varphi(x)$ :

Definition 3. A formal power series $\varphi(x)=\sum_{h \geq 0} \varphi_{h} x^{h} \in \Omega[[x]]$ is a $q$-Gevrey series (of order $s \in \mathbb{R}$ ) if the series

$$
\sum_{h \geq 0} \frac{\varphi_{h}}{q^{\frac{h(h-1)}{2}}} x^{h}
$$

is convergent.

We can state our main result:

Theorem 4. Let $\varphi(x) \in x \Omega[[x]]$ be a formal solution of the equation (21) ) and let $r \in] 0,+\infty]$ be the smallest positive slope of the Newton polygon of $\mathcal{F}_{\varphi}$. If $\frac{\partial F}{\partial w_{n}}(x, \Phi) \neq 0$, then $\varphi(x)$ is a q-Gevrey series of order $1 / r 3$

As a consequence we obtain:

\footnotetext{
${ }^{3}$ We have implicitly set $1 /+\infty=0$.
} 
Corollary 5. Let $\varphi(x) \in x \Omega[[x]]$ be a formal solution of equation (2). If $F\left(x, w_{0}, w_{1}, \ldots, w_{n}\right)$ is not identically zero, then $\varphi(x)$ is a q-Gevrey series (of some nonspecified order).

1.3. When $q$ is a parameter.... Suppose that

$$
F\left(q, x, w_{0}, \ldots, w_{n}\right) \in \mathbb{C}\left[q, x, w_{0}, \ldots, w_{n}\right],
$$

where $q$ is a parameter, and that we have a formal solution $\varphi(x)=\sum_{h \geq 0} y_{h} x^{h} \in$ $\mathbb{C}(q)[[x]], 4$ Up to equivalence, there are exactly two ultrametric norms over $\mathbb{C}(q)$ such that $q$ has a norm different than 1 . For any $f(q) \in \mathbb{C}[q]$ they are defined by

(1) $|f(q)|_{q^{-1}}=d^{-\operatorname{deg}_{q} f(x)}$;

(2) $|f(q)|_{q}=d^{\operatorname{ord}_{q} f(q)}$;

where $d \in] 0,1\left[\right.$ is a fixed real number. Of course, ||$_{q}$ and ||$_{q^{-1}}$ extend to $\mathbb{C}(q)$ by multiplicativity. Notice that $|q|_{q}=d<1$ and $|q|_{q^{-1}}=d^{-1}>1$.

Taking $\Omega$ to be the completion of $\mathbb{C}(q)$ with respect to ||$_{q}$ (resp. ||$\left._{q^{-1}}\right)$, we immediately see that Theorem 1 is a particular case of Corollary 5 and that Theorem 4 becomes:

Theorem 6. Let

$$
\frac{\partial F}{\partial w_{n}}\left(q, x, y(x), \ldots, y\left(q^{n} x\right)\right) \neq 0 .
$$

If $r \in] 0,+\infty]$ (resp. $r^{\prime} \in[-\infty, 0[$ ) is the smallest positive slope (resp. the largest negative slope) of $\mathcal{F}_{\varphi}$, then

$$
\limsup _{h \rightarrow \infty} \frac{1}{h}\left(\operatorname{deg}_{q} y_{h}-s \frac{h(h-1)}{2}\right)<+\infty, \text { with } s=1 / r,
$$

and

$$
\limsup _{h \rightarrow \infty} \frac{1}{h}\left(\operatorname{ord}_{q} y_{h}-s^{\prime} \frac{h(h-1)}{2}\right)>-\infty, \text { with } s^{\prime}=-1 / r^{\prime}
$$

\section{EXAMPLES}

2.1. Colored Jones polynomial of figure 8 knot. We consider the $q$-difference equation satisfied by the generating function of the sequence of invariants of the figure 8 knot called the colored Jones polynomials ( $c f$. [Gar04, §3]):

$$
J(q, n)=\sum_{k=0}^{n} q^{n k}\left(q^{-n-1} ; q^{-1}\right)_{k}\left(q^{-n+1} ; q\right)_{k} \in \mathbb{Z}\left[q, q^{-1}\right], \forall n \in \mathbb{N}
$$

\footnotetext{
${ }^{4}$ The results that follows are actually true when we replace $\mathbb{C}$ by any field.
} 
The series $\mathcal{J}(x)=\sum_{n \geq 0} J(q, n) x^{n} \in \mathbb{C}(q)[[x]]$ satisfies the linear $q$-difference equation

$$
\begin{aligned}
& \quad\left[q \sigma_{q}\left(q^{2}+\sigma_{q}\right)\left(q^{5}-\sigma_{q}^{2}\right)\left(1-\sigma_{q}^{2}\right)\right] y(x) \\
& -x\left[\sigma _ { q } ^ { - 1 } ( 1 + \sigma _ { q } ) \left(q^{4}+\sigma_{q}\left(q^{3}-2 q^{4}\right)+\sigma_{q}^{2}\left(-q^{3}+q^{4}-q^{5}\right)\right.\right. \\
& \left.\left.\quad+\sigma_{q}^{3}\left(-2 q^{4}+q^{5}\right)+\sigma_{q}^{4} q^{4}\right)\left(q^{5}-q^{2} \sigma_{q}^{2}\right)\left(1-\sigma_{q}\right)\right] y(x) \\
& \quad+x^{2}\left[q^{5}\left(1-\sigma_{q}\right)\left(1+\sigma_{q}\right)\left(1-q^{3} \sigma_{q}^{2}\right)\right. \\
& \left.\times\left(q^{8}+\sigma_{q}\left(q^{9}-2 q^{8}\right)-\sigma_{q}^{2}\left(-q^{7}+q^{8}-q^{9}\right)+q^{7} \sigma_{q}^{3}+q^{8} \sigma_{q}^{4}\right)\right] y(x) \\
& -x^{3}\left[q^{10} \sigma_{q}\left(1-\sigma_{q}\right)\left(1+q^{2} \sigma_{q}\right)\left(1-q^{5} \sigma_{q}^{2}\right)\right] y(x)=0 .
\end{aligned}
$$

The finite slopes of the Newton polygon are: $-1 / 2,0,1 / 2$. It is clear looking at the leading term of $J(q, n)$ that $\mathcal{J}(x)$ cannot be a converging series for the norms ||$_{q}$ and ||$_{q^{-1}}$. Therefore it follows from the Bézivin and Boutabaa theorem that

$$
\limsup _{n \rightarrow 0} \frac{1}{n}\left(\operatorname{deg}_{q} J(q, n)-2 \frac{n(n-1)}{2}\right)<+\infty
$$

and

$$
\limsup _{n \rightarrow 0} \frac{1}{n}\left(\operatorname{ord}_{q} J(q, n)+2 \frac{n(n-1)}{2}\right)>-\infty
$$

Notice that modulo the AJ conjecture ( $c f$. Gar04, §1.4]), those slopes are the same as the ones defined in $\mathrm{CCG}^{+94}$.

2.2. A $q$-deformation of the second Painlevé equation. Let us consider the nonlinear $q$-difference equation associated to the analytic funtion at $(0,1,1,1)$.5

$$
F\left(x, w_{-1}, w_{0}, w_{1}\right)=\left(w_{0}+x\right)\left(w_{0} w_{1}-1\right)\left(w_{0} w_{-1}-1\right)-q x^{2} w_{0}
$$

namely

$$
(y(x)+x)(y(x) y(q x)-1)\left(y(x) y\left(q^{-1} x\right)-1\right)-q x^{2} y(x)=0 .
$$

${ }^{5}$ This example is studied in $\left.\mathrm{KMN}^{+} 05, \S 3.5\right]$ and RGTT01 Eq.(2.55)], where many other examples can be found. 
It is a $q$-deformation of $P_{I I}$. Let $\varphi(x) \in \mathbb{C}(q)[[x]]$, with $\varphi(0)=1$, be a formal solution of equation (3). Then

$$
\begin{aligned}
\mathcal{F}_{\varphi}= & \sum_{i=-1}^{1} \frac{\partial F}{\partial w_{i}}\left(x, \varphi\left(q^{-1} x\right), \varphi(x), \varphi(q x)\right) \sigma_{q}^{i} \\
= & {[(\varphi(x)+x)(\varphi(x) \varphi(q x)-1) \varphi(x)] \sigma_{q}^{-1} } \\
+ & {\left[(\varphi(x) \varphi(q x)-1)\left(\varphi(x) \varphi\left(q^{-1} x\right)-1\right)\right.} \\
& +(\varphi(x)+x) \varphi(q x)\left(\varphi(x) \varphi\left(q^{-1} x\right)-1\right) \\
& \left.+(\varphi(x)+x)(\varphi(x) \varphi(q x)-1) \varphi\left(q^{-1} x\right)-q x^{2}\right] \sigma_{q}^{0} \\
+ & {\left[(\varphi(x)+x) \varphi(x)\left(\varphi(x) \varphi\left(q^{-1} x\right)-1\right)\right] \sigma_{q} . }
\end{aligned}
$$

A formal solution of equation (3) is give by

$$
\varphi(x)=\frac{{ }_{1} \Phi_{1}\left(0 ;-q ; q,-q^{2} x\right)}{{ }_{1} \Phi_{1}(0 ;-q ; q,-q x)}=1+\frac{q}{1+q} x+\cdots,
$$

where ${ }_{1} \Phi_{1}(0 ;-q ; q, x)$ is a basic hypergeometric series:

$$
{ }_{1} \Phi_{1}(0 ;-q ; q,-q x)=\sum_{h \geq 0} \frac{q^{h(h-1)}}{(-q ; q)_{h}(q ; q)} x_{h}^{h},
$$

and

$$
(a ; q)_{h}=(1-a)(1-a q) \ldots\left(1-a q^{h-1}\right) .
$$

A direct and straightforward calculation shows that the Newton polygon of $\mathcal{F}_{\varphi}$ is regular singular, meaning that it has only one finite horizontal slope of length 2 , plus the two vertical sides. Therefore Theorem 4 implies that the solution $\varphi(x)=$ $1+\sum_{h \geq 1} \varphi_{h} x^{h}$ considered above verifies:

$$
\limsup _{h \rightarrow \infty} \frac{1}{h} \operatorname{deg}_{q} \varphi_{h}<+\infty \text { and } \limsup _{h \rightarrow \infty} \frac{1}{h} \operatorname{ord}_{q} \varphi_{h}>-\infty .
$$

In other words, the solution $\varphi(x) \in \mathbb{C}(q)[[x]]$ is convergent for both the norm ||$_{q}$ and the norm ||$_{q^{-1}}$.

We could have also remarked that ${ }_{1} \Phi_{1}(0 ;-q ; q, x)$ is a solution of the linear equation

$$
\sigma_{q}^{-2}\left(\sigma_{q}-1\right)\left(\sigma_{q}+1\right) y(x)+q^{2} x y(x)=0,
$$

whose Newton polygon has only a horizontal finite slope. This means that ${ }_{1} \Phi_{1}(0 ;-q ; q, x)$ is convergent for both ||$_{q}$ and ||$_{q^{-1}}$, and hence that $\varphi(x)$ is also convergent.

\section{Proofs}

3.1. Proof of Theorem 4, The proof follows Mal89. It relies on the ultrametric implicit function theorem; $c f$. A'C69, Ser06, SS81.

We set $\varphi(x)=\sum_{h \geq 1} \varphi_{h} x^{h}$. For any $k \in \mathbb{N}$, let $1 . \varphi_{k}(x)=\sum_{h=0}^{k} \varphi_{h} x^{h}$;

2. $\psi(x)$ be a formal power series such that $\varphi(x)=\varphi_{k}(x)+x^{k} \psi(x)$;

3. $\Psi(x)=\left(\psi(x), \psi(q x), \ldots, \psi\left(q^{n} x\right)\right)$ and $\Phi_{k}(x)=\left(\varphi_{k}(x), \varphi_{k}(q x), \ldots, \varphi_{k}\left(q^{n} x\right)\right)$. 
Let $W=\left(w_{0}, \ldots, w_{n}\right), Z=\left(z_{0}, \ldots, z_{n}\right)$. By taking the Taylor expansion of $F(x, W+Z)$ at $W$ we obtain:

$$
F(x, W+Z)=F(x, W)+\sum_{i=0}^{n} \frac{\partial F}{\partial w_{i}}(x, W) z_{i}+\sum_{i, j=0}^{n} H_{i, j}(x, W, Z) z_{i} z_{j},
$$

where $H(x, W, Z)$ is an analytic function of $2 n+3$ variables in a neighborhood of zero. Hence we can write:

$$
\begin{aligned}
0=F(x, \Phi)= & F\left(x, \Phi_{k}(x)\right)+x^{k} \sum_{i=0}^{n} \frac{\partial F}{\partial w_{i}}\left(x, \Phi_{k}\right) q^{i k} \sigma_{q}^{i} \psi \\
& +x^{2 k} \sum_{i, j=0}^{n} H_{i, j}\left(x, \Phi_{k}(x), x^{k} \Psi(x)\right) q^{(i+j) k} \sigma_{q}^{i} \psi \sigma_{q}^{j} \psi
\end{aligned}
$$

To finish the proof we have to distinguish two cases: $r<+\infty$ and $r=+\infty$.

Case 1. $r<+\infty$. We are going to choose $k \geq \sup \left(k_{1}, k_{2}+l+1\right)$, where $k_{1}, k_{2}, l$ are constructed as follows ( $c f$. the figure below). First of all let $\left(n^{\prime}, l\right) \in \mathbb{N}^{2}$ be the point of $N_{q}\left(\mathcal{F}_{\varphi}\right)$ which verifies the two properties:

1. $l$ is the smallest real number such that $(j, l) \in N_{q}\left(\mathcal{F}_{\varphi}\right)$ for some $j \in \mathbb{R}$;

2. $n^{\prime}$ is the greatest real number such that $\left(n^{\prime}, l\right) \in N_{q}\left(\mathcal{F}_{\varphi}\right)$.

Let us consider the polynomial

$$
\mathcal{L}(T)=\sum_{i=0}^{n^{\prime}}\left[\frac{1}{x^{l}} \frac{\partial F}{\partial w_{i}}(x, \Phi)\right]_{x=0} T^{i} .
$$

We chose $k_{1}$ to be a positive integer such that for any $k \geq k_{1}$, the polynomial $\mathcal{L}(T)$ does not vanishes at $q^{k}$, and $k_{2} \geq r\left(n-n^{\prime}\right)$. Notice that for any $k \geq k_{2}+l$, the smallest positive slope of $N_{q}\left(\mathcal{F}_{\varphi_{k}}\right)$ is equal to $r$ and the point $\left(n^{\prime}, l\right)$ is the "lowest" point of $N_{q}\left(\mathcal{F}_{\varphi_{k}}\right)$ with greater abscissae.

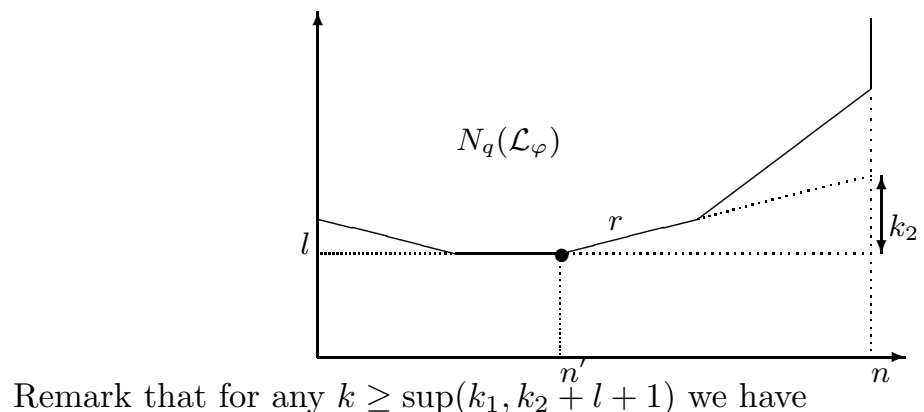

$\operatorname{ord}_{x=0} \sum_{i=0}^{n} \frac{\partial F}{\partial w_{i}}\left(x, \Phi_{k}\right) q^{i k} \sigma_{q}^{i} \psi \geq \operatorname{ord}_{x=0} \psi(x)+\inf _{i=0, \ldots, n} \operatorname{ord}_{x=0} \frac{\partial F}{\partial w_{i}}\left(x, \Phi_{k}\right) \geq l+1$.

Therefore we can write the linear part of equation (4) in the form

$$
\frac{1}{x^{l}} \sum_{i=0}^{n} \frac{\partial F}{\partial w_{i}}\left(x, \Phi_{k}\right) q^{i k} \sigma_{q}^{i} \psi=\mathcal{L}\left(q^{k} \sigma_{q}\right) \psi+x \widetilde{\mathcal{L}}\left(x, \sigma_{q}\right) \psi,
$$

where $\widetilde{\mathcal{L}}\left(x, \sigma_{q}\right)$ is an analytic functional. Moreover we deduce from equation (4) that

$$
\operatorname{ord}_{x=0} F\left(x, \Phi_{k}\right) \geq k+l+1,
$$


so that there exists an analytic function $M\left(x, w_{0}, \ldots, w_{n}\right)$ such that equation (4) divided by $x^{l+k}$ becomes

$$
\mathcal{L}\left(q^{k} \sigma_{q}\right) \psi+x \widetilde{\mathcal{L}}\left(x, \sigma_{q}\right) \psi+x M\left(x, x^{k} \Psi\right)=0 .
$$

Since $\mathcal{L}\left(q^{k} \sigma_{q}\right)$ is a linear operator with constant coefficients and $\mathcal{L}\left(q^{h}\right) \neq 0$ for any $h \geq k$, equation (5) admits one unique formal solution $\psi(x) \in x \Omega[[x]]$, whose coefficients can be constructed recursively.

In order to conclude, we have to estimate the Gevrey order of $\psi(x)$. Let us consider the following Banach $\Omega$-vector space:

$$
\mathcal{H}_{s, m}=\left\{\sum_{h \geq 1} \varphi_{h} x^{h} \in \Omega[[x]]: \sup _{h \geq 1}\left|\varphi_{h}\right||q|^{h m-s \frac{h(h-1)}{2}}<+\infty\right\}
$$

equipped with the norm

$$
\left\|\sum_{h \geq 1} \varphi_{h} x^{h}\right\|_{s, m}=\sup _{h \geq 1}\left|\varphi_{h} \| q\right|^{h m-s \frac{h(h-1)}{2}} .
$$

Since for any positive rational number $s$ and any pair of positive integers $k, h$ we have

$$
|q|^{s \frac{k(k-1)}{2}}|q|^{s \frac{k(k-1)}{2}} \leq|q|^{\frac{(k+h)(k+h-1)}{2}},
$$

the analytic functional

$$
A(\lambda, \psi)=\mathcal{L}\left(q^{k} \sigma_{q}\right) \psi+\lambda x \widetilde{\mathcal{L}}\left(\lambda x, \sigma_{q}\right) \psi+\lambda x M\left(\lambda x, \lambda^{k} x^{k} \Psi\right)
$$

is defined over $\Omega \times \mathcal{H}_{s, n^{\prime}}$ :

$$
A(\lambda, \psi): \Omega \times \mathcal{H}_{s, n^{\prime}} \longrightarrow \mathcal{H}_{s, 0},
$$

and verifies

$$
A(0,0)=0 \text { and } \frac{\partial A}{\partial \psi}(0,0)=\mathcal{L}\left(q^{k} \sigma_{q}\right) .
$$

Since $\mathcal{L}\left(q^{k} \sigma_{q}\right)$ is invertible, the implicit function theorem implies that for any $\lambda$ in a neighborhood of 0 there exists $\psi_{\lambda}$ such that $A\left(\lambda, \psi_{\lambda}\right)=0$. The formal solution $\psi$ of equation (5) being unique, we must have $\psi_{\lambda}(x)=\psi(\lambda x)$ for any $\lambda$ close to 0 , which ends the proof.

Case 2. $r=+\infty$. We chose the point $\left(n^{\prime}, l\right)$ as in the previous case: since there are no finite positive slopes, we have $n^{\prime}=n$. We can define the polynomial $\mathcal{L}(T)$ in the same way as before. So we choose $k_{1} \in \mathbb{N}$ such that $\mathcal{L}\left(q^{k}\right) \neq 0$ for any $k \geq k_{1}$ and $k_{2} \in \mathbb{N}$ such that

$$
\inf _{i=0, \ldots, n} \operatorname{ord}_{x=0}\left(\frac{\partial F}{\partial w_{i}}\left(x, \Phi_{k}\right)\right)>l
$$

for any $k \geq k_{2}$. We deduce that $\operatorname{ord}_{x=0} F\left(x, \Phi_{k}\right) \geq k+l+1$ and hence we are reduced, by dividing equation (4) by $x^{l+k}$, to consider the functional

$$
\mathcal{L}\left(q^{k} \sigma_{q}\right)+\lambda x M\left(\lambda x, \lambda^{k} x^{k} \Psi\right)=0 .
$$

The same argument as above also allows us to conclude the proof in this case. 
3.2. Proof of Corollary [5. Following Mal89, we are going to show by induction on $n$ that Theorem 4 implies Corollary 5 . Notice that for $n=0$ we are in the classical case of Puiseux development of a solution of an algebraic equation ( $c f$. Mal89]. So let us suppose $n \geq 1$.

If there exists a positive integer $k$ such that

$$
\frac{\partial^{k} F}{\partial w_{n}^{k}}(x, \Phi) \neq 0,
$$

we conclude by applying Theorem 4 to the $q$-difference equation

$$
\frac{\partial^{\kappa-1} F}{\partial w_{n}^{\kappa-1}}(x, \Phi)=0,
$$

where $\kappa$ is the smallest positive integer verifying equation (6).

We now suppose that for any positive integer $k$ we have $\frac{\partial^{k} F}{\partial w_{n}^{k}}(x, \Phi)=0$. By taking the Taylor expansion of $F\left(x, w_{0}, \ldots, w_{n}\right)$, we can verify that

$$
F\left(x, \varphi(x), \ldots, \varphi\left(q^{n-1} x\right), \psi(x)\right) \equiv 0
$$

for any

$$
\psi(x) \in x \Omega[[x]] .
$$

In particular, there exists $\lambda \in \Omega$ such that $F\left(x, w_{0}, \ldots, w_{n-1}, \lambda x\right)$ is not identically zero and $F\left(x, \varphi(x), \ldots, \varphi\left(q^{n-1} x\right), \lambda x\right)=0$. So we are reduced to the case " $n-1$ ".

\section{Complex $q$-Analog of the Maillet-Malgrange theorem for $|q|=1$}

Let $\Omega$ be either the ultrametric field defined in $\$ 1$ or the complex field $\mathbb{C}$. We choose $q \in \Omega$ such that $|q|=1$ and $q$ is not a root of unity.

To the linear $q$-difference equation

$$
\mathcal{L} y(x)=\sum_{i=0}^{n} a_{i}(x) y\left(q^{i} x\right)=0,
$$

with $a_{i}(x)=a_{i, j_{i}} x^{j_{i}}+a_{i, j_{i}+1} x^{j_{i}+1}+\cdots \in \Omega\{x\}$, we can attach a polynomial

$$
Q_{\mathcal{L}}(T)=(T-1) \sum_{i=0}^{n} a_{i, j_{i}} T^{i}
$$

We recall the result:

Theorem 7 (cf. Béz92b, Thm. 6.1] and [BB92, Thm. 6.1]). Let $\varphi(x) \in \Omega[[x]]$ be a formal solution of $\mathcal{L} y(x)=0$. We suppose that

$(\mathcal{H})$ There exist two constants $c_{1}, c_{2}>0$, such that for any root $u$ of $Q_{\mathcal{L}}(T)$ and any $n>>0$ the following inequality is satisfied: $\left|q^{n}-u\right| \geq c_{1} n^{-c_{1}}$.

Then $\varphi(x)$ is convergent.

In the nonlinear case we have the following result that generalizes [Béz92a, §1]:

Theorem 8. Let $\varphi(x) \in x \Omega[[x]]$ be a formal solution of the q-difference equation

$$
F\left(x, \varphi(x), \varphi(q x), \ldots, \varphi\left(q^{n} x\right)\right)=0,
$$

analytic at zero. We make the following assumptions:

(1) $\frac{\partial F}{\partial w_{n}}(x, \Phi) \neq 0$, 
(2) the polynomial $Q_{\mathcal{F}_{\varphi}}$ associated to the linear operator $\mathcal{F}_{\varphi}$ verifies the hypothesis $(\mathcal{H})$.

Then $\varphi(x)$ is convergent.

Remark 9. Notice that the second hypothesis is always verified in the following cases:

- if $\Omega=\mathbb{C}$ and $q$ and the coefficients of $Q$ are algebraic numbers ( $c f$. Béz92a, $2.2])$,

- if $\Omega$ is an extension of a number field $K$ equipped with a $p$-adic valuation, and $q$ and the roots of $Q(T)$ are in $K$ (in this case it is a consequence of Baker's theorem; $c f$. for instance [DV02, §8.3])

Proof of Theorem 8. The first part of the proof of Theorem 4 is completely formal. So once again we are reduced to consider equation (5)

$$
\mathcal{L}\left(q^{k} \sigma_{q}\right) \psi+x \widetilde{\mathcal{L}}\left(x, \sigma_{q}\right) \psi+x M\left(x, x^{k} \Psi\right)=0 .
$$

The key-point is the choice of $k>>1$, so that the Newton polygon of the $q$ difference operator $\mathcal{L}\left(q^{k} \sigma_{q}\right)+x \widetilde{\mathcal{L}}\left(x, \sigma_{q}\right)$ coincides with the Newton polygon of $\mathcal{F}_{\varphi}$, up to a vertical shift.

Let $\mathcal{H}(0, r)$ be the Banach algebra of analytic functions converging over the closed disk $D\left(0, r^{+}\right)$of center 0 and radius $r>0$, for $r$ small enough, equipped with the norm

$$
\left|\sum_{n \geq 0} a_{n} X^{n}\right|_{\mathcal{H}(0, r)}=\sup _{n \geq 0}\left|a_{n}\right| r^{n} .
$$

It follows from Béz92b, Thm. 6.1] and BB92, Thm. 6.16 that the operator $\mathcal{L}\left(q^{k} \sigma_{q}\right)+x \widetilde{\mathcal{L}}\left(\lambda x, \sigma_{q}\right)$ acts on $\Omega \times \mathcal{H}(0, r)$ and hence

$$
A(\lambda, \psi): \Omega \times \mathcal{H}(0, r) \longrightarrow \mathcal{H}(0, r) .
$$

The implicit function theorem also allows us to conclude this case.

Note added in proofs. Since the submission of the present paper we have obtained a stronger version of Theorem 7 in the complex case (cf. [DVz, Lemma 2.9]), which implies a stronger version of Theorem 8 . Namely, if $q=\exp (2 i \pi \tau)$, with $\tau \in$ $[0,1] \backslash \mathbb{Q}$, one can replace hypothesis $\mathcal{H}$ by the following:

$(\tilde{\mathcal{H}})$ We set $\tilde{\mathbb{Q}}_{\mathcal{L}}(T)=\sum_{i=0}^{n} a_{i, 0} T^{i}$. We suppose that $\tau$ is a Brujno number and that for any nonzero root $u \in \mathbb{C}$ of $\tilde{\mathbb{Q}}_{\mathcal{L}}(T)$ the series

$$
\sum_{m \geq 0} \frac{x^{m}}{(1-u)(1-q u) \cdots\left(1-q^{m-1} u\right)}
$$

is convergent.

\section{ACKNOWLEDGEMENT}

I would like to thank Changgui Zhang, whose questions are at the origin of this paper, and Jean-Paul Bézivin for his attentive reading of the manuscript and his numerous interesting comments.

\footnotetext{
${ }^{6}$ Notice that [BB92, Thm. 6.1] is formulated only for $q$-difference equations with polynomial coefficients, but the same proof as [Béz92b Thm. 6.1] works in the analytic case.
} 


\section{REFERENCES}

[A'C69] Norbert A'Campo. Théorème de préparation différentiable ultra-métrique. In Séminaire Delange-Pisot-Poitou: 1967/68, Théorie des Nombres, Fasc. 2, Exp. 17. Secrétariat mathématique, Paris, 1969. MR0244240 (39:5557)

[BB92] Jean-Paul Bézivin and Abdelbaki Boutabaa. Sur les équations fonctionelles $p$-adiques aux $q$-différences. Universitat de Barcelona. Collectanea Mathematica, 43(2):125-140, 1992. MR 1223416 (95j:39031)

[Béz92a] Jean-Paul Bézivin. Convergence des solutions formelles de certaines équations fonctionnelles. Aequationes Mathematicae, 44(1):84-99, 1992. MR1165786 (93d:39011)

[Béz92b] Jean-Paul Bézivin. Sur les équations fonctionnelles aux $q$-différences. Aequationes Mathematicae, 43(2-3):159-176, 1992. MR1158724 (93m:39006)

$\left[\mathrm{CCG}^{+} 94\right]$ D. Cooper, M. Culler, H. Gillet, D. D. Long, and P. B. Shalen. Plane curves associated to character varieties of 3-manifolds. Inventiones Mathematicae, 118(1):47-84, 1994. MR:1288467 (95g:57029)

[DV02] Lucia Di Vizio. Arithmetic theory of $q$-difference equations. The $q$-analogue of Grothendieck-Katz's conjecture on p-curvatures. Inventiones Mathematicae, 150(3):517-578, 2002. MR1946552(2005a:12013)

[DVz] Lucia Di Vizio. Local analytic classification of $q$-difference equations with $|q|=1$. arXiv:0802.4223

[DVRSZ03] L. Di Vizio, J.-P. Ramis, J. Sauloy, and C. Zhang. Équations aux $q$-différences. Gazette des Mathématiciens, (96):20-49, 2003. MR1988639(2004e:39023)

[FJ95] Monique Fleinert-Jensen. Théorèmes d'indices précisés et convergences des solutions pour une équation linéaire aux q-différences. Comptes Rendus de l'Académie des Sciences. Série I. Mathématique, 321(4):425-428, 1995. MR1351090 (96j:39004)

[Gar04] Stavros Garoufalidis. On the characteristic and deformation varieties of a knot. Geom. Topol. Monogr., 7:291-309, 2004. MR2172488 (2006j:57028)

$\left[\mathrm{KMN}^{+}{ }^{05}\right]$ K. Kajiwara, T. Masuda, M. Noumi, Y. Ohta, and Y. Yamada. Construction of hypergeometric solutions to the $q$-Painlevé equations. International Mathematics Research Notices, (24):1441-1463, 2005. MR2153786 (2006e:33027)

[Mai03] Edmond Maillet. Sur les séries divergentes et les équations différentielles. Annales Scientifiques de l'École Normale Supérieure. Troisième Série, 20, 1903.

[Mal89] Bernard Malgrange. Sur le théorème de Maillet. Asymptotic Analysis, 2(1):1-4, 1989. MR991413 (90f:32005)

[NM93] Fabienne Naegele (Marotte). Théorèmes d'indices pour les équations $q$-différencesdifférentielles. Comptes Rendus de l'Académie des Sciences. Série I. Mathématique, 317(6):579-582, 1993. MR1240803 (94h:34021)

[Ram78] J.-P. Ramis. Dévissage Gevrey. In Journées Singulières de Dijon (Univ. Dijon, Dijon, 1978), volume 59 of Astérisque, pages 4, 173-204. Soc. Math. France, Paris, 1978. MR:542737 (81g:34010)

[RGTT01] A. Ramani, B. Grammaticos, T. Tamizhmani, and K. M. Tamizhmani. Special function solutions of the discrete Painlevé equations. Computers 85 Mathematics with Applications. An International Journal, 42(3-5):603-614, 2001. Advances in difference equations, III. MR.1838017 (2002e:33033)

[Ser06] Jean-Pierre Serre. Lie algebras and Lie groups, volume 1500 of Lecture Notes in Mathematics. Springer-Verlag, Berlin, 2006. MR2179691 (2006e:17001)

[SSa] Yasutaka Sibuya and Steven Sperber. Convergence of power series solutions of $p$-adic nonlinear differential equation. In Recent advances in differential equations (Trieste, 1978), pages 405-419. MR643150 (83f:12021)

[SSb] Yasutaka Sibuya and Steven Sperber. Some new results on power-series solutions of algebraic differential equations. In Singular perturbations and asymptotics (Proc. Adv. Sem., Math. Res. Center, Univ. Wisconsin, Madison, Wis., 1980), volume 45 of Publ. Math. Res. Center Univ. Wisconsin, pages 379-404. MR606047|(82d:34072)

[SS81] Yasutaka Sibuya and Steven Sperber. Arithmetic properties of power series solutions of algebraic differential equations. Annals of Mathematics. Second Series, 113:111157, 1981. MR604044 (82j:12022) 
[Zha98] Changgui Zhang. Sur un théorème du type de Maillet-Malgrange pour les équations q-différences-différentielles. Asymptotic Analysis, 17(4):309-314, 1998. MR1656811 (99j:35005)

Institut de Mathématiques de Jussieu, Topologie et Géométrie algébriques, Case 7012, 2, Place Jussieu, 75251 Paris Cedex 05, France

E-mail address: divizio@math.jussieu.fr 